\title{
Sex differences in early vulnerability to cerebral injury and their neurodevelopmental implications
}

\author{
SARAH RAZ, RITA GOLDSTEIN, TRACY L. HOPKINS, MARY D. LAUTERBACH, \\ FARHANA SHAH, and CYNTHIA L. PORTER \\ University of Memphis, Memphis, Tennessee \\ W. WEBSTER RIGGS \\ University of Tennessee Medical School and Baptist Memorial Hospital-East \\ Memphis, Tennessee \\ and \\ LYNN H. MAGILL and CRAIG J. SANDER \\ Baptist Memorial Hospital-East, Memphis, Tennessee
}

\begin{abstract}
Sex differences in early vulnerability to cerebral injury were examined in a 10-year cohort of preterm infants. The 173 infants, whose gestational age was 32 weeks or less, were diagnosed with intracranial hemorrhage (ICH), the most common cerebral insult in the premature neonate. Determination of ICH severity was based on neuroimaging evidence obtained during the neonatal period. Because the females in the sample exhibited greater dysmaturity coupled with lower birthweight, as compared with their male counterparts, analysis of covariance was applied to the data. After statistically controlling for the influence of potentially confounding perinatal variables, gender was found to provide a unique contribution to extent of ventricular dilation, an index of ICH severity. Male sex was associated not only with greater ventriculomegaly, but also with a higher grade of ICH. The latter effect was demonstrable in a large subsample of 72 males and 77 females whose birthweights were $1,750 \mathrm{~g}$ or below, and who did not differ significantly in gestational age. The implications of these results for theories about the neurobiological origins of sex differences in susceptibility to deviation from normal development are addressed.
\end{abstract}

Beginning with gestation, the human male is more vulnerable to adversity than the human female. There is a higher frequency of miscarried and stillborn males (Baumgartner, Pessin, Wegman, \& Parker, 1950; McMillen, 1979; Rhodes, 1965), and the rate of neonatal survival is significantly lower in males than in females (Brothwood, Wolke, Gamsu, Benson, \& Cooper, 1986; Yu, Downe, Astbury, \& Bajuk, 1986; Yu, Wong, Bajuk, Orgill, \& Astbury, 1986). The risks for such obstetric complications as toxemia (Toivanen \& Hirvonen, 1970), placenta previa, abruptio placenta (Ounsted, 1972), premature rupture of membranes (Johnston, Sanchez-Ramos, \& Benrubi, 1989), and intrauterine growth retardation and prematurity (Villar, Khoury, Finucane, \& Delgado, 1986; McGregor, Left, Orleans, \& Baron, 1992) are also elevated in males. In addition, males are significantly less

This work was supported in part by a Center of Excellence grant from the State of Tennessee to the Department of Psychology, University of Memphis, and by a faculty research support grant awarded to the first author by Memphis State University. W. W. Riggs is associated with the departments of radiology at his two affiliations; L. H. Magill is associated with the Department of Nuclear Medicine and the Department of Radiology and C. J. Sander is on the Neonatal Intensive Care Unit at Baptist Memorial Hospital. Correspondence should be addressed to S. Raz, Department of Psychology, University of Memphis, Memphis, TN 38152. responsive to antenatal preventive treatment (Zachman, Morrison, Curet, \& Gustafson, 1989). Vulnerability persists among the survivors, as evidenced by diminished Apgar scores (Brothwood et al., 1986; Singer, Westphal, \& Niswander, 1968) and by an increased incidence of a host of perinatal complications in the male newborn. These include neurological events, such as neonatal convulsions (Taylor \& Ounsted, 1972), and pulmonary disorders-hyaline membrane disease, apnea, pulmonary infection, and pulmonary hemorrhage (Brothwood et al., 1986; Butler \& Bonham, 1963; Catlin et al., 1990; Torday, Nielsen, Fencl, \& Avery, 1981)- which may culminate in central nervous system (CNS) insult and in diminished neonatal survival (Khoury, Marks, McCarthy, \& Zaro, 1985; Perelman, Palta, Kirby, \& Farrell, 1986). The boundaries of the period during which males remain at higher morbidity risk are not established. However, manifestations of enhanced CNS sensitivity in males do persist in infancy, as suggested by an increased incidence of febrile seizures and temporal lobe epilepsy (Taylor, 1969).

A higher rate of obstetric and perinatal complications may account for the lower threshold for expression of neurodevelopmental and psychiatric disorders observed in males (Gualtieri \& Hicks, 1985). Male predominance has been reported in populations with mental retardation (Abramowicz \& Richardson, 1975; Richardson, Koller, 
\& Katz, 1987), learning disabilities (Nichols \& Chen, 1981; Tallal, 1991), language disorders (Ingram, 1959; Tallal, 1991; Robinson, 1991), autism (Bryson, Clark, \& Smith, 1988; Smalley, Asarnow, \& Spence, 1988), hyperactivity, and conduct disorders (Dykman \& Ackerman, 1991; Szatmari, Offord, \& Boyle, 1989; Trites, Dugas, Lynch, \& Gerguson, 1979). In schizophrenia, a disorder with a probable neurodevelopmental origin - suggested by an elevated rate of perinatal complications (Mednick, Parnas, \& Schulsinger, 1987) - increased severity coupled with greater changes in normal brain morphology is observed in affected males (see Raz \& Raz, 1990, for review).

In the current investigation, we sought to determine whether the extent of neonatal cerebral insult was linked to gender and, if so, whether gender effects might be due to enhanced brain vulnerability in males or to nonneurological sex differences that indirectly influence the severity of early brain damage. Intracranial hemorrhage (ICH), the most common neurologically significant lesion of the preterm infant, was used as a model of an early cerebral insult.

Intracranial hemorrhage affects up to $40 \%$ of infants who weigh less than 2,000 $\mathrm{g}$ (Shackelford \& Volpe, 1985; Volpe, 1989b). The incidence of the insult increases with the degree of prematurity (Volpe, 1989a). The hemorthage is well visualized in vivo using cranial ultrasonography. The high base rate of $\mathrm{ICH}$ in the preterm population and the availability of the neuroimaging technology suited for direct evaluation of its presence and extent create a unique opportunity for the investigation of early sex differences in CNS vulnerability.

The intracranial bleed typically begins at the subependymal germinal matrix, a richly vascularized region overlying the head of the caudate nucleus. Between 10 and 20 weeks of gestation, the matrix is the source of neuroblasts which migrate to the surface of the brain to form the cerebral cortex, and in the third trimester, it is the source of glioblasts which develop into oligodendroglia and astrocytes (Volpe, 1987, 1989a). The matrix is exhausted at term (Shackelford \& Volpe, 1985). A sequel of subependymal-intraventricular hemorrhage is destruction of the germinal matrix and, more importantly, its glial precursor cells (Volpe, 1989a). The lesion has been attributed to impaired autoregulation of blood flow and hypoxic-ischemic damage (Lou et al., 1979; Papile, Burstein, Burstein \& Koffler, 1978; Volpe, 1989a).

A germinal matrix hemorrhage may expand and subsequently rupture into the adjacent lateral ventricles, prompting a substantial increase in the volume of the ventricular system. Persistent intraventricular hemorrhage may, in turn, culminate in obstructive hydrocephalus or destruction of neighboring brain parenchyma. Papile et al. (1978) have proposed a radiological classification scheme to describe the natural history and extent of ICH. This method has subsequently been shown to have value for the purpose of outcome prediction, as discussed below. According to this system, which is now in use in many acute care centers, an isolated subependymal he- morrhage is rated as Grade I, intraventricular hemorrhage without ventricular enlargement as Grade II, intraventricular hemorrhage with ventricular enlargement as Grade III, and intraventricular hemorrhage with parenchymal extension as Grade IV.

The adverse neurodevelopmental sequelae of intracranial hemorrhage include motor disabilities such as spastic diplegia (Amato, Howald, \& von Muralt, 1986) and a general decrement in motor performance (Lewis \& Bendersky, 1989). Global cognitive deficits (Lewis \& Bendersky, 1989) and specific impairment in language and communication skills have also been reported (Bendersky \& Lewis, 1990; Brown, Bendersky, \& Chapman, 1986; Janowsky \& Nass, 1987). The likelihood and degree of impairment are directly linked to the severity (i.e., grade) of the hemorrhage (e.g., Amato et al., 1986; Miller-Sostek, Smith, Katz, \& Grant, 1987), which, in turn, reflects the probability and extent of permanent parenchymal damage.

Amato, Howald, and von Muralt (1987) reported that the incidence of intracranial hemorrhage is significantly higher in males with birthweight below $1,500 \mathrm{~g}$ than in females in the same birthweight category. They proposed that this sex difference was due to a lag in cerebral vascular maturation which rendered males more prone to CNS lesions. According to this line of reasoning, the process of involution of the germinal matrix and its fragile capillary bed - a process which ends at termmay be accelerated in females. The immature capillary network of the germinal matrix (Pinar, Edwards, Fratkin, \& Marin-Padilla, 1985) is remodeled into a mature capillary bed as the matrix disappears (Volpe, 1989a). Throughout the period of involution, a greater abundance of immature periventricular capillaries may place the preterm male at a greater risk for loss of the structural integrity of the matrix. Presently, it is unknown whether the higher incidence of ICH in males reported by Amato et al. (1987) is indeed due to maturational factors resulting in sex differences in CNS vulnerability, or whether it is the consequence of nonneurological factors which increase the probability of perinatal cerebral injury in males. In particular, the well-documented male predisposition to pulmonary complications (Catlin et al., 1990), the higher incidence of prematurity in males (McGregor et al., 1992; Villar et al., 1986), and a sex ratio which favors males at birth (Childs, Cantelino, \& Dyke, 1962) may produce an increased incidence of the lesion in males.

The study reported herein was guided by the thesis that male predilection for deviation from normal neurocognitive and neurobehavioral development might be linked to heightened CNS vulnerability to perinatal insult. The reviewed evidence on increased neurological morbidity in males provides meager support for this notion, because gender differences in the incidence of neurological disorders may be secondary to sex differences in the frequency of nonneurological obstetric or perinatal events. In the current investigation, we sought to examine whether-after taking into account antepartum, intra- 
partum, and neonatal status in the two genders-males incur a graver brain insult than do females. The severity of the lesion was indexed both by the grade (as defined by Papile et al., 1978) and by the extent of ventriculomegaly. By adjusting for confounding perinatal factors, we hoped to isolate variance in ICH severity which is attributable to early sex differences in CNS vulnerability. Such evidence would contribute to the body of knowledge on the neurobiological characteristics that place males at higher risk for deviation from normal development. The methods of the current study entailed use of neuroimaging data for direct estimation of the unique effect of gender on the extent of perinatal ICH.

\section{METHOD}

\section{Subjects}

The initial pool of subjects included all 190 infants ( 99 males) with $\mathrm{ICH}$, whose gestational age was 32 weeks or less, and who had received neonatal care at the Neonatal Intensive Care Unit (NICU) of Baptist Memorial Hospital (BMH)-East, Memphis, Tennessee, between December 1981 and October 1991. Due to the growth in NICU admissions during the mid and late $80 \mathrm{~s}$, most of these infants $(86 \%)$ were hospitalized between 1985 and 1991. The infants were diagnosed with the lesion following prospective radiological screening of all cases in this gestational age category. Most of the neonates had previously been included in a study on the lateralization of ICH (Raz et al., 1994). However, in contrast to our previous studies, the current investigation was limited to singleton births. Both surviving (defined as those who had been discharged home) and nonsurviving (defined as those who expired prior to discharge) infants were included. During this decade, the same four neonatologists provided acute care at the NICU, ensuring a high degree of continuity and uniformity in diagnostic and treatment procedures.

The cases diagnosed with ICH were initially identified through review of the discharge summaries of all infants who had been hospitalized at the NICU within the 10-year span of this investigation. The medical records (including serial radiological reports, maternal delivery records, NICU admission assessment, respiratory orders/blood gas reports, etc.) were then studied and analyzed to extract antepartum, intrapartum, and neonatal data. The information was collected from charts or microfilms under the guidance of the codirector of BMH-NICU (C.J.S.), and the supervision of the first author. The information was then thoroughly examined for errors by the first author. Consultation with C.J.S. and the pediatric radiologist (W.W.R.) was conducted when appropriate.

In the course of the study, four records from 1988, one from 1989 , six from 1990, and three from 1991 became unavailable, as charts from these years were being microfilmed. A small number of charts (one from 1985, two from 1987, and two from 1991) could not be found. Altogether, the final sample of infants for whom we were able to obtain all the relevant data included the remaining 173 infants (90 males and 83 females), 4 of whom ( 3 males) had expired during hospitalization. This sample incorporated the preponderance $(91 \%)$ of the infants who comprised our initial subject pool of 190 cases. The racial distribution within the male (69 Caucasian, 21 African-American) and female (61 Caucasian, 21 African-American) groups was similar.

\section{Scanning and Diagnostic Procedures}

At BMH-East NICU, all infants of 32 weeks gestation or less are prospectively screened with cranial ultrasonography for the presence of intracranial hemorrhage within the first 5 days of life. Because screening of infants whose gestational age is above 32 weeks is typically limited to those whose condition necessitates remaining at least a week in BMH-NICU, the sample of infants whose gestational age fell above the cutoff and who were screened for ICH is probably a biased one. Thus, our decision to restrict the study to gestation of 32 weeks or less stemmed from the obvious need to investigate sex differences in a sample that was representative of infants in a particular gestational age range.

After the first screening, the NICU infants are followed up periodically in the nursery, initially at least weekly, until discharge from the hospital. Multiple serial scans in four coronal and three sagittal planes are obtained, permitting the study of each case from the emergence of ICH to its resolution, stabilization, or until a ventriculoperitoneal shunt is placed. Prior to 1989, real-time sonographic scanning had been performed through the anterior fontanelle using a Technicare scanner with a $5-\mathrm{MHz}$ transducer. This machine was subsequently replaced by a Diasonics DRF -400 scanner and a $7.5-\mathrm{MHz}$ transducer. During the 10 -year period covered by this investigation, the majority of the evaluations were completed by the same pediatric radiologist (W.W.R.), thus providing significant diagnostic consistency. Because this investigation was carried out retrospectively, the radiologist was obviously blind to the hypotheses of the study.

The classification system proposed by Papile et al. (1978) was routinely used for diagnosis. There were 79 (41 females) infants diagnosed with Grade I, 7 (5 females) with Grade II, 68 (30 females) with Grade III, and 19 (7 females) with Grade IV hemorrhage. Hence, the distribution of hemorrhage was uneven, with $46 \%$ of the cases incurring Grade I, $4 \%$ Grade II, 39\% Grade III, and $11 \%$ Grade IV hemorrhage. Because the hemorrhage may gradually evolve over the first few days or weeks, the final grading was based on the most grave ultrasound reading obtained for the case. There were 5 infants ( 1 female) who had been diagnosed with periventricular cystic leukomalacia (PVL). It is not firmly established whether PVL is etiologically distinct or whether the pathogenesis of this severe ischemic lesion is linked to ICH (Volpe, 1989a). Thus, Papile et al.'s (1978) standard grading system was retained, and PVL was not considered in determination of lesion severity. Eight infants ( 5 males) required shunting due to severe and progressive expansion of the ventricular system. Lesion severity in these infants was coded according to Papile et al.'s conservative stratification method, which does not necessitate a separate classification for infants who need shunting.

To assess the reliability of the radiological diagnoses, we acquired a set of independent severity rankings for 10 randomly chosen cases from L.H.M., an experienced pediatric radiologist. The rankings were then correlated with those originally furnished by W.W.R. The intraclass correlation (ICC; Shrout \& Fleiss, 1979) for two raters obtained for severity diagnoses was .86. Although quite respectable, this ICC is probably an underestimate of the true inter-rater reliability for severity diagnoses, because it reflects both inter-rater agreement and stability across time. Additionally, unlike the first rater, the second one did not have the benefit of real-time imaging.

\section{Determination of Extent of Ventricular Dilation}

In addition to Papile et al.'s traditional categorical grading system, we also used radiological rankings of extent of ventriculomegaly in the analyses of sex differences that follows. The radiological ranking system of ventricular size yielded a more continuous measure of ICH severity than did Papile et al.'s method. According to the procedure used at BMH (see Raz et al., 1994), the sonogram reports include a ranking of each lateral cerebral ventricle on a 5-point system as normal, high-normal (or upper range of normal), mildly or slightly enlarged, moderately enlarged, or considerably enlarged. Because each ventricle is evaluated separately, the summation of the rankings for each lateral ventricle $(0$, nor$m a l$, to 4 , considerable) yields a 9 -point continuum $(0-8)$ on which 
the extent of lateral ventriculomegaly may be indexed. Since the infants had typically received multiple serial scans, we have chosen the most severe radiological ranking to derive an index of $\mathrm{ICH}$ severity. The most extreme right and left ventriculomegaly did not necessarily occur on the same date. However, the correlation between the radiological rankings for the right and left ventricle was very high $(r=0.91, p<.001)$. The strong association between right and left ventriculomegaly probably reflects the tendency of ICH, or the hypoxic-ischemic insult associated with it, to exert its effects on the entire ventricular system (albeit with a left-hemisphere bias, as we have reported previously; Raz et al., 1994). The 9-point index of ventriculomegaly was significantly correlated with the four grade rankings of hemorrhage severity $(r=.82 ; p<$ .001 ).

Data about the reliability of our radiological estimates of ventriculomegaly have been published elsewhere (Raz et al., 1994). Briefly, the ICC for agreement between two experienced radiologists on extent of ventriculomegaly in 9 brains (18 hemispheres) was high $(\mathrm{ICC}=.95)$, suggesting excellent reliability.

For the purpose of the current study we have also studied the concurrent validity of the radiological rankings in order to establish their relationships with a truly continuous computer-based planimetric measure of ventricular size. The scans of 18 infants (gestational age $=34.22 \pm 3.62$ weeks), whose ventricular sizes ranged from normal to considerably enlarged, were obtained prospectively. Area measures were derived from the films using the JAVA image analysis system (Jandel Scientific Corp., San Rafael, CA). The images were positioned on a light table (TrueLite 100 , NAL Consulting), and the areas of the ventricles were digitized from the parasagittal slices (two right and two left cuts) using a video camera (Cohu, high-resolution solid-state by Cohu Electronic Camera, San Diego, CA) equipped with a Fujinon TV Zoom (12.5-75 mm, F1.2) lens and a frame-grabber board (TARGA-M8, AT\&T Corp., Murray Hill, NJ). The angled parasagittal view was selected because all the components of the lateral ventricles are featured on this cut, including the frontal, occipital, and temporal horns, as well as the trigone. This view was therefore considered to provide a more valid representation of the ventricular system than would the coronal view. For analysis, the images were stored on a disk of a general-purpose desktop computer and displayed on a high-resolution 25-in. video monitor (Sony Trinitron PVM-2530). The areas of interest (AOIs) were carefully outlined by a trained operator using a digitizing tablet (Summasketch II, Summagraphics Corp.). The regions were then computed using the JAVA software. The operator, a graduate student in pediatric neuropsychology, used several neurosonographic atlases to delineate the perimeters of the lateral cerebral ventricles on the angled parasagittal cuts (Fischer, Anderson, Schuman, \& Stinton, 1985; Rumack, Horgan, Hay, \& Kindsfater, 1990; Rumack \& Johnson, 1984). In tracing the borders of the lateral ventricles, the choroid plexus was included since we had found this practice to improve interrater reliability. The temporal horns were excluded, because they did not always resolve clearly. The correlations between the radiologist's (W.W.R.) rankings of ventricular size and our morphometric measures of left and right lateral ventricular area (each averaged from two parasagittal slices) were $0.77(p<.001)$ and 0.80 $(p<.001)$ for the left and right ventricles, respectively.

\section{RESULTS}

Data about the distribution of antepartum, intrapartum, and neonatal variables in 90 males and $83 \mathrm{fe}-$ males with ICH are presented in Tables 1,2 , and 3 . Group comparisons of frequency data were accomplished via a $2 \times 2$ chi-square test with Yates correction. When cell frequency was five or less, Fisher's exact probabil-
Table 1

Summary of Obstetric Data" on Males and Females Diagnosed With Intracranial Hemorrhage

\begin{tabular}{ccc}
\hline Clinical & Males & Females \\
Characteristics & $(N=90)$ & $(N=83)$ \\
\hline
\end{tabular}

Maternal/antepartum factors:

Abruptio placenta

Age

Chorioamnionitis

Diabetes

Parity

Placenta previa

Membranes ruptured over $24 \mathrm{~h}$

Steroids ${ }^{b}$

Toxemia

$28.06(5.52)$

7

2

$0.65(0.83)$

7

16

31

12

$27.68(5.39)$

11

3

Intrapartum factors:

Abnormal presentation

Apgar score (5 min)

Apgar $<7$

Birthweight (g)

Caesarian section

Forceps delivery

General anesthesia

Gestational age $^{\mathrm{c}}$

17
$7.12(1.75)$
26
$1,351(476)$
45
10
12
$29.22(2.38)$

6

$25 \ddagger$

39

14

11 $6.99(1.58)$ $1,157(402) \dagger$ 37

9

5

aFrequencies are reported for discrete data; means and standard deviations (in parentheses) are reported for continuous data. ${ }^{b} B e t a m e t h-$ asone, to promote fetal lung maturity. 'Determined by maternal dates. ${ }^{*} p<.06 . \quad \dagger p<.01 . \quad \ddagger p<.05$.

ity test was used. The $t$ test for independent samples was employed to examine the significance of continuous data (means and standard deviations are provided in the tables).

As Table 1 shows, males and females with $\mathrm{ICH}$ were found to be similar in the frequency of maternal and antepartum risk factors such as toxemia, abruptio placenta, and placenta previa, all of which had been reported to occur more frequently in males. With the exception of prolonged rupture of membranes, a complication that tended to occur more often in females, the distribution of maternal risk factors such as age at delivery, parity, and the frequency of chorioamnionitis and diabetes were similar in the two groups. There were no significant group differences in mean 5-min Apgar score, the frequency of abnormal presentation, Cesarean sections, forceps deliveries, and use of general anesthesia. However, gender-related differences in intrapartum status did emerge in our cohort of infants. As expected, females weighed less, but they were also significantly less mature than males (see also Figure 1). Gestational age was determined by maternal dates because gestational age estimation by postnatal examination is less accurate for preterm infants (see DiPietro \& Allen, 1991, for a comprehensive review of methodological issues in gestational age estimation).

Data about respiratory assistance in the delivery room and during hospitalization are presented in Table 2 . Inspection of the table reveals no significant differences between the genders in need for intubation. Although females required an additional week of respiratory support during hospitalization, the difference did not reach conventional statistical significance. 
Table 2

Respiratory Support Data” for Males and Females With Intracranial Hemorrhage

\begin{tabular}{lccc}
\hline \multicolumn{4}{c}{ Intracranial Hemorrhage } \\
& $\begin{array}{c}\text { Males } \\
(N=90)\end{array}$ & $\begin{array}{c}\text { Females } \\
(N=83)\end{array}$ & \\
\hline Intubation required $^{\mathrm{b}}$ & 51 & 48 & n.s. \\
Ventilation $^{\mathrm{c}}$ & $10.41(15.54)$ & $14.43(19.87)$ & $p<.15$ \\
Total support $^{\mathrm{d}}$ & $21.49(27.43)$ & $28.48(33.90)$ & $p<.15$ \\
Peak inspired oxygen (\%) $^{76.21(28.86)}$ & $79.26(25.66)$ & n.s. \\
\hline
\end{tabular}

${ }^{a}$ Frequencies are reported for discrete data; means and standard deviations (in parentheses) are reported for continuous data. bendotracheal intubation needed in delivery room or immediately thereafter, upon admission to the NICU, " Number of days during which mechanical ventilation was required. 'Days of respiratory support, including mechanical ventilation, continuous positive airway pressure (CPAP), and oxyhood.

Table 3 presents information about peri- and neonatal complications which may precede, accompany, or culminate in changes in cerebral perfusion and concomitant hypoxic-ischemic cerebral insult. Such complications include metabolic/biochemical aberrations (e.g., acidosis, hypercapnia, hypocalcemia, hypoglycemia), pulmonary/ respiratory disorders (e.g., apnea, hyaline membrane disease, pneumothorax), systemic infections (sepsis), acute neurological dysfunction (seizures), and other major complications of prematurity (e.g., hypotension, patent ductus arteriosus) which signify increased neurobiological risk (Brazy, Eckerman, Oehler, Goldstein, \& O'Rand, 1991; Hill \& Volpe, 1989). Table 3 reveals that males and females did not differ in the frequency of any specific complication of prematurity, with the exception

Table 3

Sex Distribution" of Major Neonatal Complications

\begin{tabular}{|c|c|c|}
\hline Complication & $\begin{array}{c}\text { Males } \\
(N=90) \\
\end{array}$ & $\begin{array}{l}\text { Females } \\
(N=83) \\
\end{array}$ \\
\hline Anemia at birth (hematocrit $<35 \%$ ) & 10 & 3 \\
\hline Apnea & 74 & 63 \\
\hline Acidosis (initial arterial blood $\mathrm{pH}^{\mathrm{b}}$ ) & $7.26(0.12)$ & $7.26(0.11)$ \\
\hline Bronchopulmonary dysplasia (BPD) ${ }^{\mathrm{c}}$ & 23 & 26 \\
\hline Hyaline membrane disease (HMD) ${ }^{d}$ & 66 & 61 \\
\hline Hypercapnia (initial arterial $\left.\mathrm{CO}_{2}\right)^{b}$ & $45.47(13.10)$ & $44.42(11.71)$ \\
\hline Hypocalcemia $(<7 \mathrm{mg} / \mathrm{dl})$ & 56 & 52 \\
\hline Hypoglycemia (<40 mg/dl plasma) & 2 & 3 \\
\hline Hyponatremia (<130 mmol/liter) & 3 & 2 \\
\hline Hypotension ${ }^{e}$ & 19 & 25 \\
\hline Hyperbilirubinemia (12 mg/dl) & 85 & 72 \\
\hline Intrauterine growth retardation (IUGR) & 5 & 6 \\
\hline Necrotizing enterocolitis ${ }^{\mathrm{f}}$ & 6 & 9 \\
\hline Patent ductus arteriosus (PDA) ${ }^{g}$ & 20 & 21 \\
\hline Pneumonitis & 3 & 8 \\
\hline Pneumothorax & 12 & 8 \\
\hline Pulmonary hemorrhage & 3 & 5 \\
\hline Seizures & 10 & 11 \\
\hline Sepsis ${ }^{\mathrm{h}}$ & 21 & $38^{*}$ \\
\hline
\end{tabular}

${ }^{a}$ Frequencies are reported for discrete data, means and standard deviations for continuous data. 'Initial blood gas data typically obtained within one hour after birth for inborn infants. 'Radiological or clinical diagnosis. Documented by a chest roentgenogram and by clinical evaluation. ${ }^{\circ}$ Cases with low blood pressure necessitating treatment with dopamine and volume expansion. ${ }^{f}$ Documented by radiographic changes, positive stool guiacs and abdominal distention. gIdentified by clinical manifestations and echocardiographic means. hTypically documented by positive blood culture. ${ }^{*} p<.01$. of sepsis, which was significantly more common in female infants.

To examine the relationships between ICH grade (defined according to Papile et al., 1978) and sex, the four grade categories were dichotomized in order to facilitate the statistical analyses, as the number of cases with Grade II and Grade IV hemorrhage was relatively small. We have used the traditional grouping of Grade I with Grade II cases as "mild" and Grade III with Grade IV as "severe" bleeds because this dichotomy has been extensively validated. The literature on developmental outcome reveals a significant increase in neurological deficits and motor disabilities (Garfinkel et al., 1988; Morales, 1987; Williamson, Desmond, Wilson, Andrew, \& Garcia-Prats, 1982), compromised cognitive performance in infancy and school age (Leonard et al., 1990; Morales, 1987), elevated frequency of developmental articulation disorders (Garfinkel et al., 1988) and deficient perceptual motor skills (Selzer, Lindgren, \& Blackman, 1992) in children who had incurred severe bleeds as compared with those who had incurred minor bleeds.

The distribution of mild (Grades I and II) and severe (Grades III and IV) hemorrhage within the 90 males and 83 females comprising our cohort did not differ significantly ( 40 mild and 50 severe vs. 46 mild and 37 severe for males and females, respectively; $\chi^{2}=2.08 ; p<.15$, two-tailed), although the trend was in the predicted direction. In light of the significantly increased immaturity in the female cohort (see gestational age distribution by gender in Figure 1) and given the well-documented association between lower gestational age or birthweight and increased hemorrhage severity (Perlman \& Volpe, 1986), even a nonsignificant trend for the relatively mature group (i.e., males) to incur more severe cerebral bleeds is remarkable. Conversely, even given a comparable degree of ICH in the two genders, a significant sex difference in the extent of maturity at birth-in favor of males - can hardly be expected. Yet such a difference is consistent with the maturational lag hypothesis of increased male vulnerability to ICH (Amato et al., 1987), suggesting that the extent of neurological vulnerability in preterm males resembles that observed in preterm females who are significantly less mature.

A proper comparison of ICH severity in the two sexes requires that they be somehow equated on gestational age (and other perinatal variables). This may be accomplished statistically, using analysis of covariance (ANCOVA), or by imposing a restriction that will produce groups that are comparable on the variable(s) of interest (e.g., gestational age). To illustrate the latter point, we restricted the sample to infants below $1,750 \mathrm{~g}$. This resulted in the elimination of only 24 infants from the sample. With the exception of sepsis that was more common in females $\left(\chi^{2}=5.05, p<.05\right)$, the remaining 72 males and 77 females did not differ significantly on any pre-, peri-, or postnatal variable, including gestational age (mean $\pm S D=28.64 \pm 2.29$ weeks and $28.17 \pm 2.65$ weeks for males and females, respectively; $t=1.16$, n.s.), although the females continued to exhibit the expected 


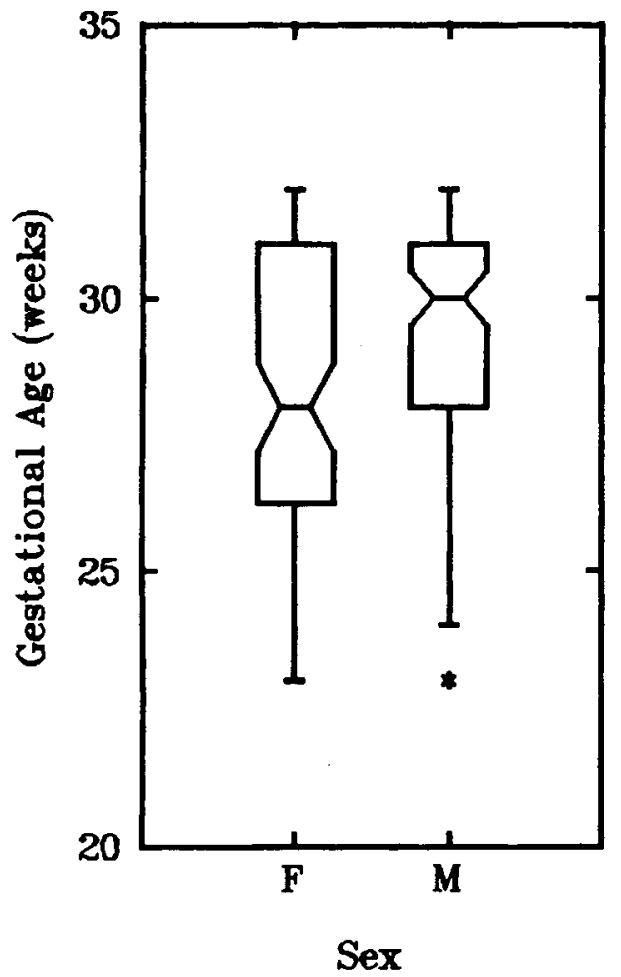

Figure 1. A box plot illustrating the distribution of gestational age for both sexes. Each box incorporates the interquartile range of the values with the horizontal line in the middle indicating the median and the whiskers demarcating the values within 1.5 interquartile range from the median. A single value marked by a star is an outlier whose gestational age was below these limits. The width of the notch in the boxes is proportional to $95 \%$ confidence limits around the median.

trend for lower birthweight (mean $\pm S D=1,175 \pm 307 \mathrm{~g}$ and $1,092 \pm 315 \mathrm{~g}$ for males and females, respectively; $t=1.62, p<.11)$. After obtaining two groups that were roughly similar in perinatal status, examination of the distribution of mild vs. severe hemorrhage within the sexes revealed a significant difference. In this large subsample $(N=149)$ from our original cohort of 173 infants, there were 26 males with mild ICH ( 24 with Grade I, 2 with Grade II) and 46 males with severe ICH ( 35 with Grade III, 11 with Grade IV), versus 41 females with mild hemorrhage ( 36 with Grade I, 5 with Grade II) and 36 females with severe hemorrhage (29 with Grade III, 7 with Grade IV), yielding a chi-square of $4.42(p<.05)$. In accord with the hypotheses of the study, this distribution suggested enhanced male vulnerability to severe ICH.

To assess the unique effects of gender on severity of perinatal cerebral insult in the context of multiple perinatal risk factors, the data on the entire cohort of 173 infants, for whom we were able to obtain a complete set of the relevant background variables, were subjected to an ANCOVA. In this analysis, the variable of interest, sex, was used as a grouping factor. The dependent variable was the 9-point index of extent of ventriculomegalydetermined from the radiological rankings-as de- scribed in the method section. Seven variables were introduced as covariates to control for the potentially significant contribution of perinatal risk factors. These included birthweight, Apgar score at $5 \mathrm{~min}$, initial arterial blood $\mathrm{pH}$ value (degree of acidosis), number of days on which respiratory assistance was required, and peak oxygen requirement during hospitalization. The latter two variables are indices of degree of respiratory distress. Because the relative contribution of obstetric and neonatal risk factors to severity of CNS insult is unestablished, simple summary scores of 9 obstetric complications (abruptio placenta and previa, chorioamnionitis, toxemia, breech and forceps delivery, use of general anesthesia, premature rupture of membranes, and maternal diabetes) and 16 neonatal complications were introduced as covariates. The neonatal complications list incorporated all medical conditions recorded in Table 3, with several exceptions. Intrauterine growth retardation (IUGR; defined as birth weight below the 10th percentile for gestational age according to growth charts provided in Battaglia \& Lubchenco, 1967) was excluded because it overlaps with both birthweight and gestational age. Also excluded were neonatal seizures, the probable sequel of either the hemorrhage itself or the hypoxic-ischemic insult that had led to its occurrence, and acidosis. The latter was entered separately into the regression model because of its well-established independent association with ICH (Beverley \& Chance, 1984; Levene, Fawer, \& Lamont, 1982; Weindling et al., 1985).

To reduce multicollinearity, gestational age, a variable closely linked to birthweight $(r=.78)$, was not entered as a covariate. Birthweight was preferred to gestational age because it reflects variance associated with both gestational age and sex, and because in our sample gestational age was artificially truncated at 32 weeks. Ventilation days were highly correlated with days of respiratory support $(r=.81)$. The latter variable included days in which oxygen supplementation without ventilatory assistance was required. Respiratory support was preferred to ventilation days as a covariate because it reflected a broader range of treatment needs and was therefore expected to account for a larger share of variance in ICH severity. Finally, degree of hypercapnia (carbon dioxide retention) was not entered into the equation because it shared a substantial amount of variance with acidosis $(r=-.76)$. The correlations between the seven covariates (perinatal risk factors) and the 9-point index of ventriculomegaly were $r=.29, .25, .20, .37$, $.31, .02$, and .37 for birthweight, Apgar score at $5 \mathrm{~min}$, initial $\mathrm{pH}$, total respiratory support, highest percent of oxygen required, number of obstetric complications, and number of neonatal complications. All correlations, with the exception of the number of obstetric complications, were significant $(p<.01)$.

The interactions between the covariates and sex were initially included in the full model to test the assumption of homogeneity of regression slopes. These interaction terms were discarded after estimation of the full model revealed that they were not significant. The subsequent 
analyses revealed that the model without the interactions accounted for $23 \%$ of the variance in the predicted variable. Consistent with the hypotheses of this study, sex provided a significant contribution to explaining variance in extent of ventriculomegaly $[F(1,164)=5.31$, $p<.05]$, with males exhibiting greater dilation than females. From the seven covariates, only birthweight and initial $\mathrm{pH}$ accounted for a significant proportion of the variance $[F(1,164)=4.55$, and $F(1,164)=4.87$, respectively, $p<.05]$. Lower birth weight and increased acidosis were linked to greater ventriculomegaly. Repeating the same ANCOVA procedure with gestational age substituted for birthweight yielded similar results. The effect of sex on extent of ventriculomegaly remained significant $[F(1,164)=4.35, p<.05]$. The relation between extent of ventriculomegaly and sex is depicted in Figure 2.

Analyses of ICH lateralization in our subjects replicated our previous results in a sample of 148 infants (Raz et al., in press), most of whom were included in the current study. In the cohort of 173 singletons, 119 infants ( 55 females) suffered bilateral hemorrhage and 54 were diagnosed with unilateral bleeds. In the latter group, there were 39 subjects (18 males) with left- and 15 subjects ( 8 males) with right-hemisphere hemorrhage. Hence, there were significantly more left than right bleed diagnoses $\left(\chi^{2}=10.66, p<.01\right)$, but, as we have previously reported, no sex differences could be demon-

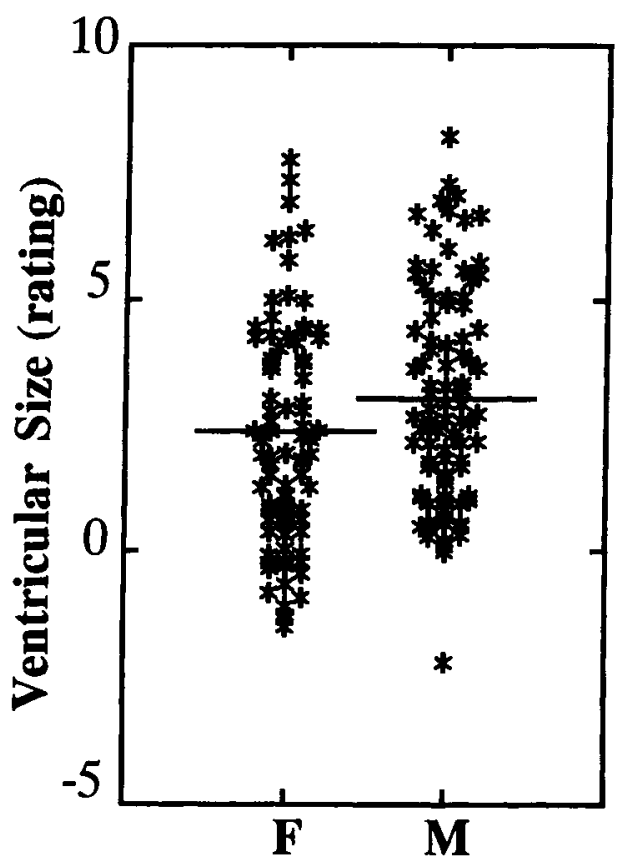

Figure 2. Extent of ventriculomegaly adjusted for initial pH (acidosis), birthweight, 5-min Apgar score, days of respiratory support, highest percent of oxygen required, number of obstetric complications, and number of neonatal complications in a cohort of 173 preterm neonates. Horizontal lines correspond to adjusted means. Note that due to statistical adjustment for perinatal status several cases have negative values. strated in lesion lateralization $\left(\chi^{2}=0.22\right.$, n.s. $)$. Additionally, no sex differences were found in the occurrence of uni- versus bilateral bleeds ( 26 unilateral and 64 bilateral vs. 28 unilateral and 55 bilateral in males and females, respectively; $\chi^{2}=.27$, n.s.). The latter finding is hardly surprising since unilateral bleeds tend to occur primarily in the milder cases of ICH $(83 \%$ of the unilateral cases in our sample were ranked as Grade I or Grade II, and not a single unilateral case with Grade IV could be found). Hence, although bilateral bleed is more severe than unilateral bleed, leading one to expect a higher frequency of such hemorrhage in males, the chances of finding sex differences within a restricted range of severity were nonetheless small. At this time, however, it is unknown whether a broader range of severity would have yielded different results.

\section{DISCUSSION}

On the basis of evidence obtained via pediatric neuroimaging, we have found gender to be a significant contributor to the severity of early cerebral insult in a cohort of premature newborns. In a sample of 149 infants with birthweights of $1,750 \mathrm{~g}$ or less, males were shown to incur significantly more severe intracranial bleeds than females, although the genders did not differ significantly on any pre-, peri-, or postnatal risk factor, with the exception of sepsis. When the effects of confounding perinatal factors favoring males (e.g., higher gestational age) were statistically adjusted for, gender effects in ICH severity favoring females could be demonstrated in the entire cohort of 173 infants. The traditional ranking of hemorrhage severity (Papile et al., 1978) was used in the former (nonparametric) analysis, whereas extent of ventriculomegaly was employed as the predicted variable in the latter (multivariate) analysis.

There are probably hundreds of antepartum, intrapartum, and postpartum variables which might contribute to enhanced male vulnerability to cerebral injury. In particular, gender effects in the severity of CNS lesions may be attributable to group differences in the frequency of obstetric and neonatal complications that had been reported to occur more often in males. Yet, in our sample, we were unable to detect any differences in antepartum, intrapartum, or neonatal status in favor of females. The absence of the expected female advantage in perinatal risk is apparently due to the composition of our sample which included only infants with cerebral lesions. Within this select group, it is unlikely that sex differences which had been demonstrated on entire cohorts would be found. If anything, our cohort of females tended to have a more disadvantageous antepartum, intrapartum, and neonatal clinical course, as suggested by a significantly lower gestational age, a higher incidence of sepsis, and a trend for a higher incidence of prolonged rupture of membranes. The latter complication is associated with elevated risk for newborn infections, but it is also linked to reduced risk for respiratory difficulties due to accelerated fetal lung maturation (Dabiri, 1979). 
In our cohort, we could not document any beneficial effects of prolonged rupture of membranes. The 25 female newborns whose mothers had experienced this complication did not differ significantly in peri- or neonatal clinical status from the 58 females whose mothers had had intact membranes $24 \mathrm{~h}$ prior to delivery. The variables on which the two groups were compared included Apgar score at $5 \min (t=1.15$, n.s. $)$ and three indices of respiratory difficulties-number of days in which ventilatory support was needed, days of respiratory assistance, and peak oxygen required $(t=-0.02,0.29$, and -0.14 , respectively, all n.s.).

It is plausible that dissimilar neonatal treatment procedures might yield sex differences such as those observed here. Indeed, it is difficult, if not impossible, to quantify all the differences in treatment procedures which may have occurred among infants during a prolonged hospitalization. However, we believe it unlikely that systematic differences in medical treatment between male and female infants have occurred in our cohort of neonates, notwithstanding the fact that females required a somewhat longer intervention period-including ventilatory support - apparently due to their increased prematurity. The greater requirements of female infants for intervention would only be expected to offset the observed gender differences in vulnerability to cerebral insult. Because the sex ratio in our sample did not change significantly from year to year $\left[\chi^{2}(10)=.59\right.$, n.s.], it is also unlikely that time-related changes in diagnostic or therapeutic technology have affected the genders differentially.

The racial distribution was similar in both groups, but, due to the archival nature of our study, we were unable to examine the distribution of socioeconomic status (SES). However, in a previous investigation of a similar sample, SES effects on lesion severity could not be found (Miller-Sostek et al., 1987). We believe it unlikely that undocumented obstetric complications have yielded the sex differences found here, since such factors have been shown to play a minor, if any, role in the occurrence of ICH (Casaer, de Vries, \& Marlow, 1991).

The origins of male vulnerability are yet to be established. It has been proposed that early sex differences in susceptibility to disease may be rooted in different rates of maturation between the genders. Males presumably lag behind females, thus remaining increasingly prone to morbidity and mortality during a given gestational or postnatal period (Gualtieri \& Hicks, 1985; Taylor, 1969; Taylor \& Ounsted, 1972). The hypothesized maturational lag, estimated from about 3 (Taylor, 1985) to 6 weeks (Garai \& Scheinfeld, 1968) at birth, may manifest itself in retarded development of extracerebral as well as intracerebral structures. For instance, on the basis of the observation of increasing vulnerability to seizures with declining age, Taylor (1969) hypothesized that the greater incidence of temporal lobe epilepsy in male infants implied slowed (temporal lobe) maturation. Retarded maturation of extracerebral structures in males is exemplified by delay in skeletal (Roche, 1968) and pul- monary development (Catlin et al., 1990; Nielsen, Torday, Fencl, \& Avery, 1979).

Our findings are consistent with the notion of retarded maturation in males (Geschwind \& Galaburda, 1985; Taylor, 1969). If, indeed, perinatal sex differences in cerebral lesion severity are due to asynchronies in the rates of development, the question remains as to whether the greater vulnerability in males is due to neurological or to nonneurological factors. Diminished pulmonary maturity in males (Catlin et al., 1990), for instance, may lead to a greater hypoxic-ischemic insult and a subsequent exacerbation of ICH (Volpe, 1989a, 1989b). Yet, in our cohort of neonates, the percentage of males who developed respiratory distress and who required ventilatory assistance was similar to that of females. The percentage of males who developed pulmonary disorders was also similar to that of females. Two indices of degree of respiratory distress - number of mechanical ventilation days and peak oxygen requirement-also did not disclose male disadvantage. Undocumented changes in cerebral perfusion resulting from respiratory distress and its concomitant metabolic derangements are probably largely accounted for by the extent of ventilatory support and, in the present sample, would be expected to occur more frequently in females. Thus, although the possibility cannot be excluded, our findings do not support the notion that diminished pulmonary maturity and accompanying respiratory difficulties are major contributors to the sex differences observed in the severity of $\mathrm{ICH}$.

In all likelihood, neurobiological factors may account for male predisposition to a graver course of early cerebral lesions. The hypothesized acceleration of CNS development in females may provide some protection against exacerbation of $\mathrm{ICH}$. One may speculate, for instance, that the specific mechanism underlying enhanced female resilience to germinal matrix injury involves accelerated involution of the germinal matrix and its fragile capillary bed. The diminished capillary bed, in turn, may render females less likely than males to bleed profusely, and, as noted by Volpe (1989a), it is the amount of intraventricular blood that ultimately determines the severity of the lesion.

It should be emphasized that different rates of maturation between the sexes need not be postulated to account for gender differences in perinatal vulnerability to cerebral hemorrhage. Cerebral oxygen requirements are higher in males than in females (Hutt, 1972), and males may be more susceptible to ischemia due to reduced elasticity of blood vessels (Cope \& Roach, 1977). In animal studies, heightened susceptibility of male rats to experimentally induced transient ischemia has been demonstrated (Woodward, Downes, Lessov, Williams, \& Meshul, 1990). Thus, regardless of developmental factors, the male brain may possess structural or physiological properties that produce increased perinatal vulnerability to hypoxia-ischemia and their sequel, cerebral injury.

In their review of the literature on "selective male affliction," Gualtieri and Hicks (1985) concluded that the 
pattern of sex differences is such that although females are less susceptible to neurodevelopmental disorder, they are more prone to a severe course when afflicted. This conclusion is based on reports of increased severity of developmental disorders such as autism, mental retardation, and hyperactivity in females. The neurobiological sex differences that produce the dissociation between the elements of frequency and severity of developmental disorder observed by Gualtieri and Hicks (1985) are yet to be established. Our results imply that the greater incidence of males with developmental disorder could be linked, at least in part, to enhanced perinatal vulnerability to cerebral insult. Increased predisposition to early CNS damage would be expected to result in a larger number of males who have crossed the neurobiological threshold toward a diagnosable neurological, cognitive, or psychiatric disorder. The poorer neurodevelopmental outcome in females, on the other hand, may not be attributable to an elevated risk for a graver perinatal CNS injury but perhaps, as suggested by Gualtieri and Hicks (1985), to genetic factors. In the studied cohort of preterm infants, females suffered a milder cerebral insult despite their disadvantageous perinatal course. To further elucidate the putative perinatal sources of sex differences in susceptibility to neurodevelopmental disorder, it is essential that the cognitive and behavioral outcome of males and females with a similar extent of neurobiological risk be studied. With the availability of noninvasive pediatric neuroimaging, the genders may be matched for extent of early CNS insult, and this issue can be examined.

\section{REFERENCES}

Abramowicz, H. K., \& Richardson, S. A. (1975). Epidemiology of severe mental retardation in children: Community studies. American Journal of Mental Deficiency, 80, 18-39.

amato, M., Howald, H., \& von Muralt, G. (1986). Neurological prognosis of high risk preterm infants with peri-intraventricular hemorthage and ventricular dilatation. European Neurology, 25, 241-247.

amato, M., Howald, H., \& von Muralt, G. (1987). Fetal sex and distribution of peri-intraventricular hemorrhage in preterm infants. European Neurology, 27, 20-23.

BAtTAGLia, F. C., \& LuBChEnCo, L. O. (1967). A practical classification of newborn infants by weight and gestational age. Journal of Pediatrics, 71, 159-163.

Baumgartner, L., Pessin, V., Wegman, M., \& Parker, S. (1950). Weight in relation to fetal and newborn mortality: Influence of sex and color. Pediatrics, 6, 329-342.

BENDERSKY, M., \& LEWIS, M. (1990). Early language ability as a function of ventricular dilatation associated with intraventricular hemorrhage. Developmental \& Behavioral Pediatrics, 11, 17-21.

Beverley, D. W., \& Chance, G. (1984). Cord blood gases, birth asphyxia and intraventricular hemorrhage. Archives of Diseases in Childhood, 59, 884-897.

Brazy, J. E., Eckerman, C. O., Oehler, J. M., Goldstein, R. F., \& O'RAND, A. M. (1991). Nursery neurobiologic risk score: Important factors in predicting outcome in very low birth weight infants. Journal of Pediatrics, 118, 783-792.

Brothwood, M., Wolke, D., Gamsu, H., Benson, J., \& CoOPER, D. (1986). Prognosis of the very low birthweight baby in relation to gender. Archives of Disease in Childhood, 61, 559-564.

Brown, B. B., Bendersky, M., \& ChapMAN, T. (1986). The early utterances of preterm infants. British Journal of Disorders of Communication, 21, 307-319.
Bryson, S. E., Clark, B. S., \& SMITH, I. M. (1988). First report of a Canadian epidemiological study of autistic syndromes. Journal of Child Psychology \& Psychiatry, 29, 433-455.

Butler, N. R., \& Bonham, D. G. (1963). Perinatal mortality. Edinburgh: Churchill Livingstone.

Casaer, P., de Vries, L., \& Marlow, N. (1991). Prenatal and perinatal risk factors for psychosocial development. In M. Rutter \& P. Casaer (Eds.), Biological risk factors for psychosocial disorders (pp. 139174). Cambridge: Cambridge University Press.

Catlin, E. A., Powell, S. M., Manganaro, T. F., Hudson, P. L., Ragin, R. C., Epstein, J., \& Donahoe, P. K. (1990). Sex-specific fetal lung development and Muellerian inhibiting substance. American Review of Respiratory Diseases, 141, 466-470.

Childs, B., Cantoline, S., \& Dyke, M. D. (1962). Observations on sex differences in human biology. Bulletin of the Johns Hopkins Hospital, 110, 134-144.

COPE, D., \& ROACH, M. (1977). Effects of high static pressures on human cerebral arteries in vitro. Stroke, 8, 254-257.

DABIRI, C. (1979). Respiratory distress syndrome. In T. Field, A. MillerSostek, S. Goldberg, \& H. H. Shuman (Eds.), Infants born at risk (pp. 251-259). New York: SP Medical and Scientific Books.

DiPIETRO, J. A., \& ALLEN, M. C. (1991). Estimation of gestational age: Implications for developmental research. Child Development, 62 , 1184-1199.

Dykman, R. A., \& Ackerman, P. T. (1991). Attention deficit disorder and specific reading disability: Separate but often overlapping disorders. Journal of Learning Disabilities, 24, 96-103.

Fischer, Q. A., Anderson, J. C., Schuman, R. M., \& Stinton, W. (1985). Pediatric neurosonography: Clinical, tomographic and neuropathologic correlates. New York: Wiley.

Garai, J. E., \& SCHEINFELd, S. (1968). Sex differences in mental and behavioral traits. Genetic Psychology Monographs, 77, 169-299.

Garfinkel, E., Tejani, N., Boxer, H. S., Levinthal, C., Atluru, V., Tuck, S., \& Vidyasagar, S. (1988). Infancy and early childhood follow-up of neonates with periventricular or intraventricular hemorrhage or isolated ventricular dilation: A case controlled study. American Journal of Perinatology, 5, 214-219.

Geschwind, N., \& GaLABURDA, A. M. (1985). Cerebral lateralization: Biological mechanisms, associations, and pathology: II. A hypothesis and a program of research. Archives of Neurology, 42, 521-552.

GuAltieri, T., \& Hicks, R. (1985). An immunoreactive theory of selective male affliction. Behavioral \& Brain Sciences, 8, 427-441.

HiLl, A., \& Volpe, J. J. (1989). Hypoxic-ischemic encephalopathy of the newborn. In K. Swaiman (Ed.), Pediatric neurology (pp. 373392). St. Louis: Mosby

HuTt, C. (1972). Neuroendocrinological, behavioral, and intellectual aspects of sexual differentiation in human development. In C. Ounsted \& D. C. Taylor (Eds.), Gender differences, their ontogeny and significance (pp. 73-121). Edinburgh: Churchill Livingstone.

INGRAM, T. T. S. (1959). Specific developmental disorders of speech in childhood. Brain, 82, 450-467.

JANOWSKY, J. S., \& NASs, R. (1987). Early language development in infants with cortical and subcortical perinatal brain injury. Behavioral \& Developmental Pediatrics, 8, 3-7.

Johnston, M., Sanchez-Ramos, L., \& Benrubi, G. I. (1989). Premature rupture of membranes prior to 34 weeks gestational age: One year experience at a tertiary center. Journal of Florida Medical Association, 76, 767-771.

Khoury, M. J., Marks, J. S., McCARThy, B. J., \& ZARo, S. M. (1985). Factors affecting the sex differential in neonatal mortality: The role of respiratory distress syndrome. American Journal of Obstetrics \& Gynecology, 151, 777-782.

Leonard, C. H., Clyman, R. I., Piecuch, R. E., Juster, R. P., BalLARD, R. A., \& BOOTH BEHLE, M. (1990). Effect of medical and social risk factors on outcome of prematurity and very low birthweight. Journal of Pediatrics, 116, 620-626.

LEVENE, M. I., FAwER, C. L., \& LAMONT, R. F. (1982). Risk factors in the development of intraventricular hemorrhage in the preterm neonate. Archives of Diseases in Childhood, 57, 410-417.

LEWIS, M., \& BENDERSKY, M. (1989). Cognitive and motor differences among low birth weight infants: Impact of intraventricular hemorrhage, medical risk, and social class. Pediatrics, 83, 187-192. 
Lou, H. C., Lassen, N. A., Tweed, W. A., Johnson, G., Jones, M., \& Palaniuk, R. J. (1979). Pressure passive cerebral blood flow and breakdown of the blood-brain barrier in experimental fetal asphyxia. Acta Paediatrica Scandinavica, 68, 57-63.

MCGregor, J. A., LeFT, M., Orleans, M., \& Baron, A. (1992). Fetal gender differences in preterm birth: Findings in a North American cohort. American Journal of Perinatology, 9, 43-48.

MCMilleN, M. M. (1979). Differential mortality by sex in fetal and neonatal deaths. Science, 204, 89-91.

Mednick, S. A., Parnas, J., \& Schulsinger, F. (1987). The Copenhagen high-risk project, 1962-86. Schizophrenia Bulletin, 13, 485495.

Miller-Sostek, A., Smith, Y. F, Katz, K. S., \& Grant, E. G. (1987). Developmental outcome of preterm infants with intraventricular hemorrhage at one and two years of age. Child Development, 58, 779786.

MORALES, W. J. (1987). Effect of intraventricular hemorrhage on the one-year mental and neurologic handicaps of the very low birth weight infant. Obstetrics \& Gynecology, 70, 111-114.

Nichols, P. L., \& CHEN, T. C. (1981). Minimal brain dysfunction: A prospective study. Hillsdale, NJ: Erlbaum.

Nielsen, H. C., Torday, J. S., Fencl, M., \& Avery, M. E. (1979). Sex differences in human fetal lung maturation. Pediatric Research, 13, 361.

Ounsted, M. (1972). Gender and intrauterine growth. In C. Ounsted \& D. C. Taylor (Eds.), Gender differences, their ontogeny and significance (pp. 177-201). Edinburgh: Churchill Livingstone.

Papile, L. A., Burstein, J., Burstein, R., \& Koffler, H. (1978). Incidence and evolution of the subependymal and intraventricular hemorrhage: A study of infants with birth weights less than $1,500 \mathrm{gm}$. Journal of Pediatrics, 92, 529-534.

Perelman, R. H., Palta, M., Kirby, R., \& Farrell, P. M. (1986). Discordance between male and female deaths due to the respiratory distress syndrome. Pediatrics, 78, 238-244.

Perlman, J. M., \& VolPE, J. J. (1986). Intraventricular hemorrhage in extremely small premature infants. Archives of Diseases in Childhood, 140, 1122-1125.

Pinar, M. H., Edwards, W. H., Fratkin, J., \& Marin-Padilla, M. (1985). A transmission electron microscopy study of human cerebral cortical and germinal matrix (GM) blood vessels in premature neonates. Pediatric Research, 19, 394A.

Raz, S., Foster, M. S., Briggs, S. D., Shah, F., Baertschi, J. C., Lau'terbach, M. D., Riggs, W. W., Magill, L. H., \& Sander, C. J. (1994). Lateralization of perinatal cerebral insult and cognitive asymmetry: Evidence from neuroimaging. Neuropsychology, 8, 160-170.

RAZ, S., \& RAZ, N. (1990). Structural brain abnormalities in the major psychoses: A quantitative review of the evidence from computerized imaging. Psychological Bulletin, 108, 93-108.

RHODES, P. (1965). Sex of the fetus in ante partum hemorrhage. Lancet, 2, 718-719.

Richardson, S. A., Koller, H., \& Katz, M. (1987). Sex differences in the classification of children as mildly mentally retarded. Uppsala Journal of Medical Science, 44 (Supplement), 83-88.

RoBINSON, R. J. (1991). Causes and associations of severe and persistent specific speech and language disorders in children. Developmental Medicine \& Child Neurology, 33, 943-962.

Roche, A. F. (1968). Sex-associated differences in skeletal maturity. Acta Anatomica, 71, 321-340.

Rumack, C. M., Horgan, J., Hay, T. C., \& Kindsfater, D. (1990). Pocket atlas of pediatric ultrasound. New York: Raven Press.

Rumack, C. M., \& Johnson, M. L. (1984). Perinatal and infant brain imaging: Role of ultrasound and computed tomography. Chicago: Yearbook Medical.

Selzer, S. C., Lindgren, S., \& Blackman, J. A. (1992). Long-term neuropsychological outcome of high risk infants with intracranial hemorrhage. Journal of Pediatric Psychology, 17, 407-422.
SHACKELFORD, G. D., \& VOLPE, J. J. (1985). Cranial ultrasonography in the evaluation of neonatal intracranial hemorrhage and its complications. Journal of Perinatal Medicine, 13, 293-304.

Shrout, P. E., \& FLEISs, J. L. (1979). Intraclass correlations: Uses in assessing raters' reliability. Psychological Bulletin, 86, 420 428.

Singer, J. E., WestPhal, M., \& Niswander, K. R. (1968). Sex differences in the incidence of neonatal abnormalities and abnormal performance in early childhood. Child Development, 30, 103-1 12.

Smalley, S. L., Asarnow, R. F., \& SpEnce, M. A. (1988). Autism and genetics: A decade of research. Archives of General Psychiatry, 45 953-961.

SzatMari, P., OfFord, D. R., \& Boyle, M. H. (1989). Ontario child health study: Prevalence of attention deficit disorder with hyperactivity. Journal of Child Psychology \& Psychiatry, 30, 219-230.

TALLAL, P. (1991). Hormonal influences in developmental learning disabilities. Psychoneuroendocrinology, 16, 203-211.

TAYLOR, D. C. (1969). Differential rates of cerebral maturation between sexes and between hemispheres. Lancet, 19, 140-142.

TAYLOR, D. C. (1985). Development rate is the major differentiator between the sexes. Behavioral \& Brain Sciences, 8, 459-460.

TAYLOR, D. C., \& OUNSTED, C. (1972). The nature of gender differences explored through ontogenetic analyses of sex ratios in disease. In C. Ounsted \& D. C. Taylor (Eds.), Gender differences: Their ontogeny and significance (176-202). Edinburgh: Churchill Livingstone.

Torvanen, P., \& Hirvonen, T. (1970). Sex ratio of newborns: Preponderance of males in toxemia of pregnancy. Science, 170, 187-188.

Torday, J. S., Nielsen, H. C., Fencl, M., \& Avery, M. E. (1981). Sex differences in fetal lung maturation. American Review of Respiratory Diseases, 123, 205-208.

Trites, R. L., Dugas, E., Lynch, G., \& Gerguson, H. B. (1979). Prevalence of hyperactivity. Journal of Pediatric Psychology, 4, 179-188.

Villar, J., Khoury, M. J., Finucane, F. F., \& Delgado, H. L. (1986). Differences in the epidemiology of prematurity and intrauterine growth retardation. Early Human Development, 14, 307-320.

VoLPE, J. J. (1987). Neurology of the newborn. Philadelphia: Saunders.

VoLPE, J. J. (1989a). Intraventricular hemorrhage in the premature infant - current concepts. Part I. Annals of Neurology, 25, 3-11.

VoLPE, J. J. (1989b). Intraventricular hemorrhage in the premature infant-current concepts. Part II. Annals of Neurology, 25, 109-116.

Weindling, A. M., Wilkinson, A. R., CoOK, J., Calvert, S. A., Fok, T. F., \& ROCHEForT, M. J. (1985). Perinatal events which precede periventricular haemorrhage and leukomalacia in the newborn. British Journal of Obstetrics \& Gynaecology, 92, 1218-1223.

Williamson, W. D., Desmond, M. M., Wilson, G. S., ANDREW, L., \& GarCIA-PRATS, J. A. (1982). Early neurodevelopmental outcome of low birth weight infants surviving neonatal int raventricular hemorrhage. Journal of Perinatal Medicine, 10, 34-41.

WoodWARD, W. R., Downes, H., Lessov, N., Williams, T., \& Meshul, C. K. (1990). Gender-related differences and glial changes in a rat model of transient forebrain ischemia. Society for Neuroscience Abstracts, 16, 934.

Yu, V. Y., Downe, L., Astbury, J., \& Bajuk, B. (1986). Perinatal factors and adverse outcome in extremely low birthweight infants. Archives of Disease in Childhood, 61, 554-558.

Yu, V. Y., Wong, P. Y., Bajuk, B., Orglll, A. A., \& Astbury, J. (1986). Outcome of extremely low birthweight infants. British Journal of Obstetrics \& Gynaecology, 93, 162-170.

Zachman, R. D., Morrison, J. C., Curet, L. B., \& Gustafson, N. (1989). Lecithin: Sphingomyelin ratio in the amniotic fluid of male and female fetuses. Journal of Reproductive Medicine, 34, 203206.

(Manuscript received September 8, 1993; revision accepted for publication April 26, 1994.) 